Minh-Tuan Nguyen-Thai, Paul Wulff, Nils Gräbner, Utz von Wagner

\title{
On the influence of external stochastic excitation on linear oscillators with subcritical self-excitation and gyroscopic influence with application to brake squeal
}

Journal article | Accepted manuscript (Postprint)

This version is available at https://doi.org/10.14279/depositonce-10216

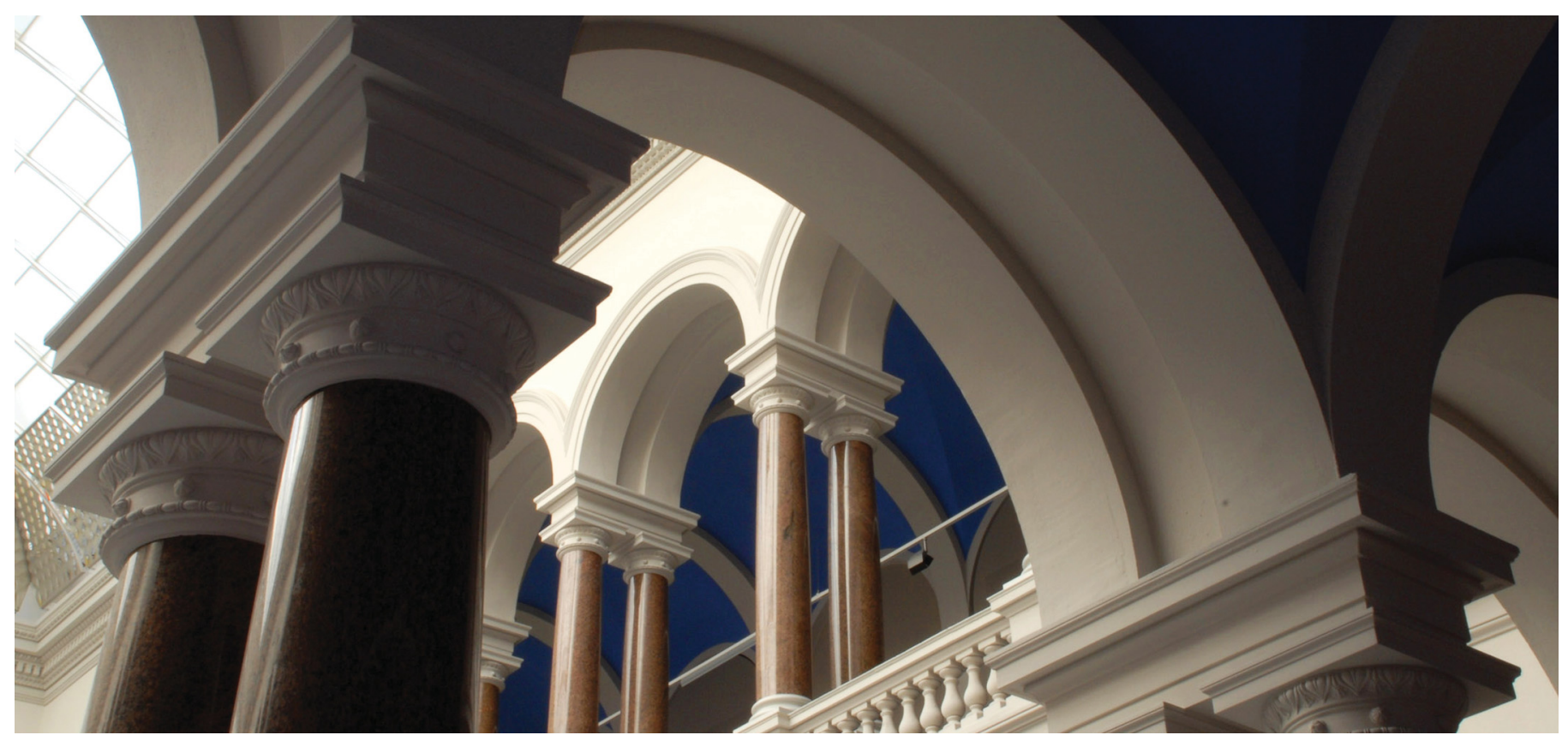

This is the peer reviewed version of the following article:

Nguyen-Thai, Minh-Tuan; Wulff, Paul; Gräbner, Nils; Wagner, Utz von (2020). On the influence of external stochastic excitation on linear oscillators with subcritical self-excitation and gyroscopic influence with application to brake squeal. ZAMM - Journal of Applied Mathematics and Mechanics.

https://doi.org/10.1002/zamm.202000113

which has been published in final form at https://doi.org/10.1002/zamm.202000113. This article may be used for non-commercial purposes in accordance with Wiley Terms and Conditions for Use of SelfArchived Versions. 


\title{
On the influence of external stochastic excitation on linear oscillators with subcritical self-excitation and gyroscopic influence with application to brake squeal
}

\author{
Minh-Tuan Nguyen-Thai*1 | Paul Wulff ${ }^{2}$ | Nils Gräbner ${ }^{2}$ | Utz von Wagner ${ }^{2}$
}

${ }^{1}$ Department of Applied Mechanics, Hanoi University of Science and Technology, Hanoi, Vietnam

${ }^{2}$ Chair of Mechatronics and Machine Dynamics, Technische Universität Berlin, Berlin, Germany

\section{Correspondence}

*Minh-Tuan Nguyen-Thai, Email: tuan.nguyenthaiminh@hust.edu.vn

\begin{abstract}
Summary
In a linear analysis for brake squeal, an unwanted type of sound in the kHz-range produced during the braking process of vehicles, usually only the stability of the system is examined. However, with the appearance of additional stochastic excitation, the vibration of a linear system with subcritical self-excitation, i.e. having self-excitation but due to damping still an asymptotically stable trivial solution, may be large enough to produce a squeal sound. In this paper, this hypothesis of stochastically reinforced self-excitation is supported by a case study on a wobbling disk model for brake squeal, which includes both circulatory and gyroscopic forces. For this example, the Fokker-Planck equation is solved and numerical integrations are performed. A short parameter study is carried out to examine the effect of damping and gyroscopic terms on these stochastically reinforced self-excitation. The results suggest that this possibility should be considered additionally to classical explanations of brake squeal.
\end{abstract}

KEYWORDS:

brake squeal, stochastic excitation, stochastically reinforced self-excitation, gyroscopic effects

\section{1 | INTRODUCTION}

This special issue paper is the subsequent study of our report on a new hypothesis on the mechanism of brake squeal at the DSTA2019 Conference in Lodz ${ }^{[1]}$ extending the therein examined linear model with gyroscopic terms and considering a minimal model for brake squeal.

Brake squeal is an unwanted type of sound in the kHz-range produced during the braking process of vehicles. Despite the existence of much literature discussing the cause and mechanism of brake squeal like the review papers $\stackrel{[2.3]}{\underline{2}}$, there has not been any mathematical model for brake squeal that fits every realistic observation and could be used for a predictive design. Since in experimental observations, the frequency of brake squeal is almost independent on disk speed, this type of noise is commonly assumed to be the result of self-excited vibration, not forced vibration. This assumption has led to many linear models with selfexcitation for brake squeal ${ }^{[4.5 .6]}$. Stability analysis is applied to these models to predict, when the unwanted vibration occurs. However, when the trivial solution of a linear system is unstable, the vibration amplitude will not be restricted and will increase to infinity as time goes on. This is not true in the reality of brake squeal, where the vibration displacement amplitudes of brake disk and the brake pads lie in the micrometer-range. Thus, nonlinear models are derived to explain the limitation of the amplitudes, and brake squeal is modeled as a limit cycle ${ }^{[7.8 .9]}$. It is extremely hard to measure the nonlinear characteristics, especially those 
of the frictional contacts ${ }^{[10,11,12]}$, so that the validities of the nonlinear models are hard to confirm. Normally, when the trivial solution of a linear system is stable, brake squeal is considered not to happen, disregarding the transient process - the period of time the system changes from an initial state to the steady state. The transient process is usually neglected because due to the negative maximum real part of the eigenvalues, unwanted phenomena are not considered to be likely to happen during this process and even if they happen, they are not expected to last long. However, if transient growth occurs and resets repeatedly, it will affect the behavior of the system significantly. Transient growth is a phenomenon in which a growth in perturbation happens at the beginning of the transient process before the decay ending in the steady state. Transient growth is well-known in turbulence studies ${ }^{[13,14]}$, but, to the knowledge of the authors, there has been only few publications in the field of mechanical engineering $\stackrel{[15,16,17]}{ }$. In $\stackrel{[1]}{ }$, we presented a two degree of freedom (2-DOF) system that can undergo repeatedly transient growths. The idea and results are summarized as follows.

Firstly, a set of homogenous linear ordinary differential equations (ODE) is considered

$$
\ddot{\mathbf{x}}+\mathbf{D} \dot{\mathbf{x}}+(\mathbf{K}+\mathbf{N}) \mathbf{x}=\mathbf{0},
$$

where $\mathbf{D}$ is the symmetric positive definite damping matrix and $\mathbf{K}$ the symmetric and also positive definite stiffness matrix. The $\mathbf{N}$ matrix is skew-symmetric and implies the appearance of non-conservative circulatory forces, which means there is possibility for the system to exhibit self-excited vibration. Here, we consider parameter cases where the trivial fixed point is asymptotically stable, thus the term "subcritical self-excitation" is used.

Then, to model uncertainties that may affect the real system, a stochastic excitation is added and Eq. (1) becomes

$$
\ddot{\mathbf{X}}_{t}+\mathbf{D} \dot{\mathbf{X}}_{t}+(\mathbf{K}+\mathbf{N}) \mathbf{X}_{t}=\boldsymbol{\sigma} \xi_{t}
$$

The expectation is that the stochastic excitation may continuously restart the transient process, leading to repeatedly transient growths that maintain over time a large enough, but bounded, vibration considered as squeal sound. In this paper, this cause of vibration is called stochastically reinforced self-excitation, since the transient growth is probably the consequence of the (subcritical) self-excitation. To confirm this effect, we consider two systems: system (2) and a respective system without circulatory forces, i.e. no subcritical self-excitation, but having the same negative maximum real part of the eigenvalues. It turns out that under an appropriate stochastic excitation, the vibration level of the system with subcritical self-excitation is much larger than that in the no self-excitation case as described in $\stackrel{[1]}{\underline{1}}$.

This paper extends these results by adding a $\mathbf{G}$ matrix, representing the appearance of gyroscopic forces, to the considered equations. Such equations can be derived from a minimal model for brake squeal with a wobbling disk.

Note that a linear model is usually the result of linearization from an original nonlinear model. In brake squeal, there is the possibility that a stable limit cycle co-exists with a stable equilibrium but also stable limit cycles and unstable equilibria as well as solely stable equilibria occur depending on parameters $\underline{[7]}, \underline{[12]}, \underline{[18]}$. In simplified considerations, the equations linearized with respect to the equilibrium are investigated and asymptotic stability of the equilibrium is interpreted as non-existence of squeal, which is in the nonlinear case only true if the basin of attraction of this equilibrium is not left. One may expect that small initial conditions (i.e. small deviations from equilibirum) are in the basin of attraction of the stable equlibrium; however, transient growth according to self-excitation and without the help of stochastic excitation is proved to make a solution even with small initial conditions able to finally reach a stable limit cycle $\stackrel{[16]}{ }$. The actual paper describes that even in the case of nonleaving the basin of attraction of the stable equilibrium, vibrations comparable to squeal may occur in the presence of external noise. Nevertheless, it is possible that stochastically reinforced self-excitation may also bring the solution to the attraction of a coexisting stable limit cycle in the nonlinear case. However, this problem is out of the scope of this paper and may be investigated in the future.

\section{2 | A 2-DOF EDGKN SYSTEM WITH STOCHASTIC EXCITATION FOR BRAKE SQUEAL}

Consider a low-DOF brake system model with wobbling disk and frictional point contacts (Fig. 1). This model includes a rigid wobbling disk and a pair of massless pads elastically suspended along both normal (associated with out-plane vibration) and circumferential (associated with in-plane vibration) directions with respect to the disk ${ }^{[12]}$. This model is an extension of the minimal model introduced in $[\underline{[}]$ while such an extension with massless pads performing circumferential movement can also already be found e.g. in $\stackrel{[19,20]}{ }$. A more complicated pad suspension system based on ${ }^{[6]}$ is found in ${ }^{[8]}$. 


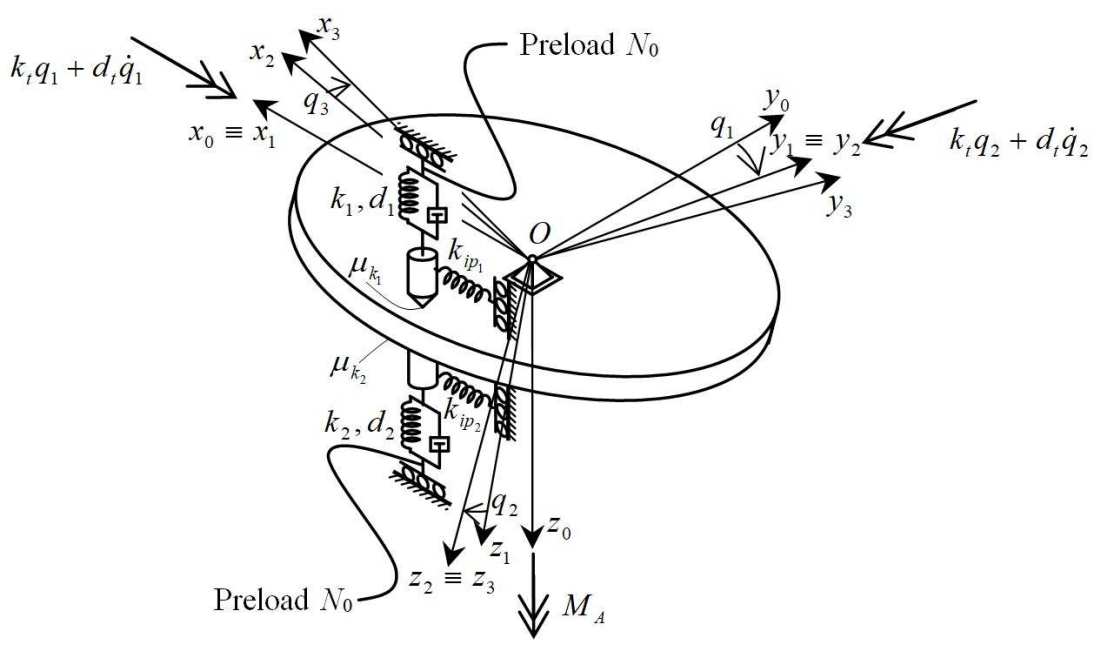

FIGURE 1 A low-DOF brake system model with wobbling disk and frictional point contacts (Fig.5.1- $\underline{[12]}$ ).

The perturbation equations are derived in $\stackrel{[12]}{ }$ and read

$$
\overline{\mathbf{M}} \ddot{\mathbf{x}}+\overline{\mathbf{B}} \dot{\mathbf{x}}+\overline{\mathbf{C}} \mathbf{x}=\mathbf{0}
$$

where

$$
\begin{gathered}
\overline{\mathbf{M}}=\left[\begin{array}{cc}
\Theta & 0 \\
0 & \Theta
\end{array}\right], \\
\overline{\mathbf{B}}=\left[\begin{array}{cc}
d_{t}+\left(d_{1}+d_{2}\right) r^{2}+\frac{\mu_{k}^{2} N_{0} h}{2}\left(\frac{d_{1}}{k_{i p_{1}}}+\frac{d_{2}}{k_{i p_{2}}}\right) & \Phi \Omega_{d} \\
\mu_{k} N_{0} r\left(\frac{d_{1}}{k_{i p_{1}}}+\frac{d_{2}}{k_{i p_{2}}}\right)-\left(d_{1}+d_{2}\right) \frac{\mu_{k} h r}{2}-\Phi \Omega_{d} & d_{t}
\end{array}\right], \\
\overline{\mathbf{C}}=\left[\begin{array}{cc}
k_{t}+N_{0} h+\left(k_{1}+k_{2}\right) r^{2}+\frac{\mu_{k}^{2} N_{0} h}{2}\left(\frac{k_{1}}{k_{i p_{1}}}+\frac{k_{2}}{k_{i p_{2}}}\right) & \frac{\mu_{k}^{2} N_{0} h}{2}\left(h-\left(1+\mu_{k}^{2}\right) \frac{N_{0}}{k_{i p_{1}}}+\frac{N_{0}}{k_{i p_{2}}}\right) \\
-\mu_{k} r\left(2 N_{0}-\frac{N_{0} k_{1}}{k_{i p_{1}}}-\frac{N_{0} k_{2}}{k_{i p_{2}}}+\frac{k_{1} h}{2}+\frac{k_{2} h}{2}\right) & k_{t}+\left(1+\mu_{k}^{2}\right) N_{0}\left(h-\frac{N_{0}}{k_{i p_{1}}}-\frac{N_{0}}{k_{i p_{2}}}\right)
\end{array}\right] .
\end{gathered}
$$

In the equations, $h$ is the thickness of the brake disk and $r$ is the effective braking radius. $\Theta$ and $\Phi$ are the moments of inertia of the brake disk with respect to its in-plane and out-of-plane symmetry axes, respectively. The disk is elastically supported by two rotational springs of stiffness $k_{t}$ and rotational dampers of damping coefficient $d_{t}$. The pads are supported in the normal direction with respective to the disk by linear springs of stiffnesses $k_{1}$ and $k_{2}$, and linear dampers of damping coefficients $d_{1}$ and $d_{2}$. The in-plane supports of the pads are linear springs of stiffnesses $k_{i p_{1}}$ and $k_{i p_{2}}$. The coefficient of kinetic friction between the pads and the disk is $\mu_{k}$ and $\Omega_{d}$ is the present macroscopic disk angular velocity, which is hereafter called in short as disk speed.

Eq. (3) can be rewritten as

$$
\ddot{\mathbf{x}}+\mathbf{B} \dot{\mathbf{x}}+\mathbf{C x}=\mathbf{0}
$$

where

$$
\mathbf{B}=\frac{1}{\Theta} \overline{\mathbf{B}} \quad, \quad \mathbf{C}=\frac{1}{\Theta} \overline{\mathbf{C}}
$$


Matrix $\mathbf{B}$ can be split into a symmetric matrix $\mathbf{D}$ (damping matrix) and a skew-symmetric matrix $\mathbf{G}$ (gyroscopic matrix), while matrix $\mathbf{C}$ can be split into a symmetric matrix $\mathbf{K}$ (stiffness matrix) and a skew-symmetric matrix $\mathbf{N}$ (circulatory matrix). Eq. (7) then reads

$$
\ddot{\mathbf{x}}+(\mathbf{D}+\mathbf{G}) \dot{\mathbf{x}}+(\mathbf{K}+\mathbf{N}) \mathbf{x}=\mathbf{0}
$$

where

$$
\begin{aligned}
& \mathbf{D}=\frac{1}{2}\left(\mathbf{B}+\mathbf{B}^{\top}\right) \quad, \quad \mathbf{G}=\frac{1}{2}\left(\mathbf{B}-\mathbf{B}^{\top}\right), \\
& \mathbf{K}=\frac{1}{2}\left(\mathbf{C}+\mathbf{C}^{\top}\right) \quad, \quad \mathbf{N}=\frac{1}{2}\left(\mathbf{C}-\mathbf{C}^{\top}\right),
\end{aligned}
$$

System (9) is correspondingly called an EDGKN system, a special case of an MDGKN system [21]. The replacement of M by the identity matrix E eases the below-mentioned parameter study. It also marks the fact that the original mass matrix in equation (3) is just a scaled identity matrix. Not all 2-DOF linear models for brake squeal have this property, e.g. the model described in ${ }^{[4]}$. The gyroscopic term also does not appear for some other models $\stackrel{[4.5]}{a}$ and the equations of motion just form an MDKN system.

It is already known that, depending on the parameters, the considered system can be either unstable, so that self-excited vibration predominates the damping, or stable, i.e. the self-excitation is subcritical [1] ${ }^{\underline{1}}$. This fact makes the system a candidate to test the abovementioned hypothesis. For stochastically reinforced self-excitation to occur, an external stochastic excitation is added to the model, whose equations then read as follows

$$
\ddot{\mathbf{X}}_{t}+(\mathbf{D}+\mathbf{G}) \dot{\mathbf{X}}_{t}+(\mathbf{K}+\mathbf{N}) \mathbf{X}_{t}=\boldsymbol{\sigma} \xi_{t}
$$

There are several candidates for this random excitation, e.g. the roughness of the contact surfaces, the frictional behavior or other sources from the surrounding. For simplicity for these fundamental investigations, the noise $\xi_{t}$ is assumed to be a scalar Gaussian white noise with zero mean and the vector $\boldsymbol{\sigma}$ contains their intensity coefficients.

\section{3 | COMPARISON OF SELF-EXCITED AND NON-SELF-EXCITED SYSTEMS IN THE PRESENCE OF STOCHASTIC EXCITATION}

To carve out the difference in the behavior of the EDGKN system and a suitable EDK system, we consider the following case. The considered EDGKN system has subcritical self-excitation, while the EDK system has no self-excitation, provided that the stiffness and damping matrices are both positive definite. If under the same stochastic excitation, the vibration largeness of the EDGKN system is much higher than that of the EDK system, it might be because the stochastic excitation effectively reinforces the self-excitation. However, this statement is not reasonable if the EDGKN system has a higher negative maximum real part of the eigenvalues than the EDK system, because in that case one may claim that this difference might be the reason for the difference in the vibration largeness. This is one reason why the two systems within one comparison should have the same negative maximum real part of the eigenvalues. The systems that share the same maximum real part of the eigenvalues are hereafter called maximum-real-part-of-the-eigenvalues-companions (MRECs). Another reason to focus on the maximum real part of the eigenvalues is to prove that the sign of the maximum real part of the eigenvalues should not be the only concern when considering linear mathematical brake squeal models.

Therefore we consider an EDGKN system, and a corresponding EDK system that has the same stiffness matrix and the damping matrix is scaled from that of the EDGKN system by

$$
\ddot{\mathbf{x}}+\alpha \mathbf{D} \dot{\mathbf{x}}+\mathbf{K x}=\mathbf{0} .
$$

Adjusting the damping scale factor $\alpha$, one might get a non-circulatory non-gyroscopic MREC of the given EDGKN system, i.e. they have the same (negative) maximum real part of the eigenvalues. Adding stochastic excitation also in this case yields

$$
\ddot{\mathbf{X}}_{t}+\alpha \mathbf{D} \dot{\mathbf{X}}_{t}+\mathbf{K} \mathbf{X}_{t}=\boldsymbol{\sigma} \xi_{t}
$$


The systems from (12) and (14) are in the following called stochastically excited maximum-real-part-of-the-eigenvaluescompanions (SEMRECs).

As a measure for the intensity of vibrations we consider the marginal probability density function (PDF) of state variables. The larger the marginal PDF deviates from zero, the larger the intensity of the vibration of the corresponding system is.

The probability density function can either be calculated using numerical integration (Monte-Carlo simulation) or by solving the corresponding Fokker-Planck equation which is in general a challenging task, but here comparably simple, as we have linear systems with Gaussian excitation. In both cases, the second-order SDEs (12) and (14) are rewritten as first-order systems of the form

$$
\mathrm{d} \mathbf{Q}_{t}=\mathbf{A} \mathbf{Q}_{t}+\mathbf{g d} W_{t}
$$

where $\mathbf{Q}_{t}$ is the vector of the random state processes

$$
\mathbf{Q}_{t}=\left[\begin{array}{c}
\mathbf{X}_{t} \\
\dot{\mathbf{X}}_{t}
\end{array}\right]
$$

$W_{t}$ is the Wiener process corresponding to $\xi_{t}$, and the other matricies are determined as follows

$$
\begin{gathered}
\mathbf{A}=\left[\begin{array}{cc}
\mathbf{0} & \mathbf{E} \\
-\mathbf{C} & -\mathbf{B}
\end{array}\right], \\
\mathbf{g}=\left[\begin{array}{l}
0 \\
0 \\
\boldsymbol{\sigma}
\end{array}\right] .
\end{gathered}
$$

The $\mathbf{B}$ and $\mathbf{C}$ matrices in both EDGKN and EDK cases can be found by rewriting the system in the form of equation (7).

The diffusion matrix is defined as

$$
\mathbf{H}=\mathbf{g g}^{\top} \text {. }
$$

The PDF $p(\mathbf{q})$ of the stationary process $\mathbf{Q}_{t}$ can be found by solving the stationary Fokker-Planck equation associated with Eq. (12) and (14)

$$
\sum_{i=1}^{4} \frac{\partial}{\partial q_{i}}\left[p(\mathbf{q}) \sum_{j=1}^{4} a_{i j} q_{j}\right]-\frac{1}{2} \sum_{i=1}^{4} \sum_{j=1}^{4} \frac{\partial^{2}}{\partial q_{i} \partial q_{j}}\left[p(\mathbf{q}) h_{i j}\right]=0,
$$

where $a_{i j}$ and $h_{i j}$ are the elements in row $i$ and column $j$ of matrix $\mathbf{A}$ and matrix $\mathbf{H}$, respectively.

Since we have a linear system with Gaussian excitation, the corresponding solution is also Gaussian. Following ${ }^{[22]}$, the solution has the form

$$
p(\mathbf{q})=\frac{1}{(2 \pi)^{2}|\mathbf{\Lambda}|^{\frac{1}{2}}} \exp \left(-\frac{1}{2} \mathbf{q}^{\top} \boldsymbol{\Lambda}^{-1} \mathbf{q}\right),
$$

with mean value zero vector and covariance matrix $\Lambda$. The mean value vector is a zero vector while the covariance matrix is to be found. Eq. (21) yields to the following algebraic equation [23]

$$
[(\mathbf{E} \otimes \mathbf{A})+(\mathbf{A} \otimes \mathbf{E})] \operatorname{vec}(\mathbf{\Lambda})+\operatorname{vec}(\mathbf{H})=0,
$$

where $\otimes$ denotes the Kronecker product and $\operatorname{vec}(\cdot)$ denotes vectorization operator.

Solving (22) for $\boldsymbol{\Lambda}$ and computing (21), one obtains $p(\mathbf{q})$. Marginal PDFs $p_{X_{1}}$ and $p_{X_{2}}$ are also Gaussian and can be calculated by 


$$
\begin{aligned}
& p_{X_{1}}\left(x_{1}\right)=p_{Q_{1}}\left(q_{1}\right)=\int_{-\infty}^{\infty} \int_{-\infty}^{\infty} \int_{-\infty}^{\infty} p(\mathbf{q}) \mathrm{d} q_{2} \mathrm{~d} q_{3} \mathrm{~d} q_{4}, \\
& p_{X_{2}}\left(x_{2}\right)=p_{Q_{2}}\left(q_{2}\right)=\int_{-\infty}^{\infty} \int_{-\infty}^{\infty} \int_{-\infty}^{\infty} p(\mathbf{q}) \mathrm{d} q_{1} \mathrm{~d} q_{3} \mathrm{~d} q_{4} .
\end{aligned}
$$

The corresponding standard deviations of the marginal PDFs can be determined as

$$
\sigma_{1}=\sqrt{\Lambda_{11}} \quad, \quad \sigma_{2}=\sqrt{\Lambda_{22}},
$$

where $\Lambda_{11}$ and $\Lambda_{22}$ are the first two element on the main diagonal of the covariance matrix $\Lambda$.

The stochastic-excitation ratios between the SEMRECs are defined as

$$
\rho_{1}=\frac{\sigma_{1-E D G K N}}{\sigma_{1-E D K}} \quad, \quad \rho_{2}=\frac{\sigma_{2-E D G K N}}{\sigma_{2-E D K}} .
$$

If either $\rho_{1}$ or $\rho_{2}$ or both are significantly higher than 1 , a stochastically reinforced self-excitation is considered to be effective.

\section{4 | COMPARISON OF VIBRATIONAL BEHAVIOR OF EDGKN AND EDK SYSTEMS SUBJECTED TO EXTERNAL STOCHASTIC EXCITATION}

\subsection{A case study}

Consider the SDE (12) that is derived from the perturbation equation (3). The parameters are chosen as follows

$$
\begin{gathered}
h=0.05 \mathrm{~m} ; \quad r=0.13 \mathrm{~m} ; \quad \Theta=0.16 \mathrm{~kg} \mathrm{~m}^{2} ; \quad \Phi=2 \Theta ; \quad k_{t}=1.88 \times 10^{7} \mathrm{Nm} ; \quad d_{t}=0.1 \mathrm{Nm} \mathrm{s} ; \\
k_{1}=6 \times 10^{6} \mathrm{Nm} ; \quad k_{2}=6 \times 10^{6} \mathrm{Nm} ; \quad d_{1}=5 \mathrm{Ns} / \mathrm{m} ; \quad d_{2}=5 \mathrm{Ns} / \mathrm{m} ; \quad k_{i p_{1}}=6 \times 10^{6} \mathrm{Nm} ; \\
k_{i p_{2}}=6 \times 10^{6} \mathrm{Nm} ; \quad \mu_{k}=0.6 ; \quad N_{0}=3000 \mathrm{~N} ; \quad \Omega_{d}=5 \pi \mathrm{rad} / \mathrm{s}
\end{gathered}
$$

Most of the physical parameters are taken from the literature ${ }^{[6,12]}$. The obtained system matrices are

$$
\begin{gathered}
\mathbf{D}=\left[\begin{array}{cc}
8.5682 & -0.059719 \\
-0.059719 & 0.625
\end{array}\right] \mathrm{s}^{-1}, \\
\mathbf{G}=\left[\begin{array}{cc}
0 & 31.476 \\
-31.476 & 0
\end{array}\right] \mathrm{s}^{-1}, \\
\mathbf{K}=\left[\begin{array}{cc}
1.1876 \times 10^{8} & -7.3072 \times 10^{4} \\
-7.3072 \times 10^{8} & 1.175 \times 10^{8}
\end{array}\right] \mathrm{s}^{-2}, \\
\mathbf{N}=\left[\begin{array}{cc}
0 & 7.3178 \times 10^{4} \\
-7.3178 \times 10^{4} & 0
\end{array}\right] \mathrm{s}^{-2} .
\end{gathered}
$$

The corresponding trivial solution is stable with the maximum real part of the eigenvalues $\lambda=-2.1451 \mathrm{~s}^{-1}$. The EDK MREC of the form (13) is obtained with the damping scale factor $\alpha=6.6933$. Though the two MRECs are both stable, their behaviors are different given the same initial condition $x=[1,0]^{\top} \mathrm{rad}$ : the EDK system shows decaying vibration with a short transient process (Fig. 2b) while the EDGKN shows a beating phenomenon and maintains much higher amplitudes during the same period (Fig. 2a). Nevertheless, conventional linear analysis for brake squeal treats them equally according to the same maximum real part of the eigenvalues. The beating phenomenon has been found also in EDKN system $\underline{[15]}$. 


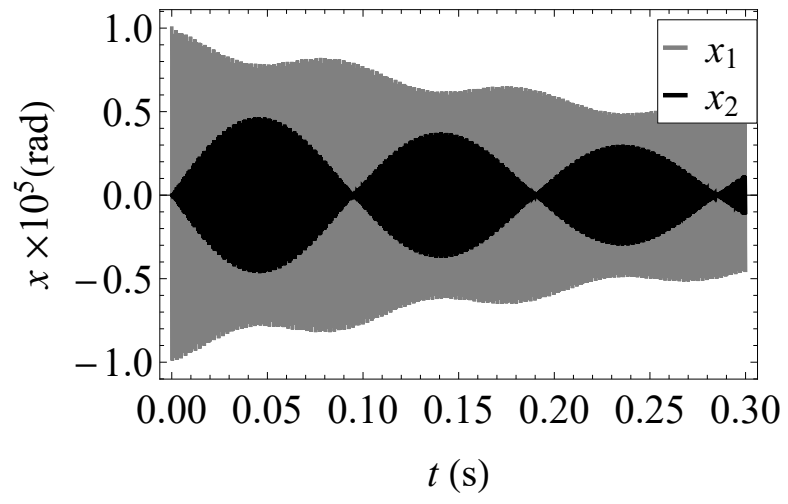

a) EDGKN system: Beating phenomenon

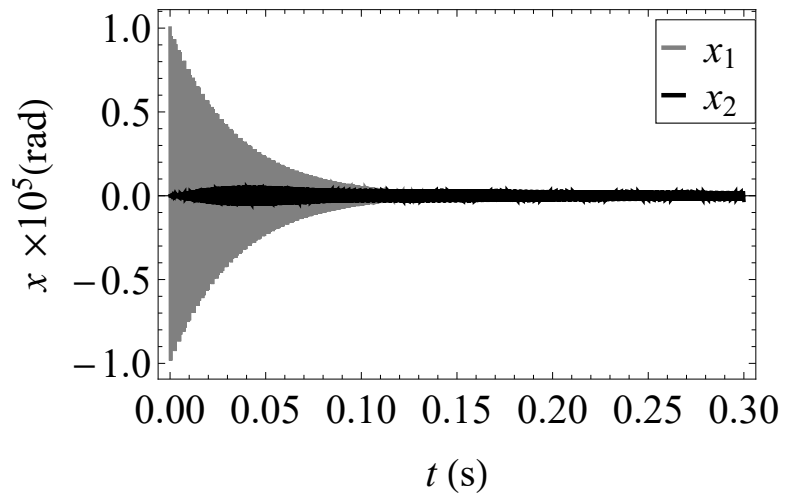

b) EDK system: Decaying vibration without beating

FIGURE 2 Time history for the deterministic MRECs.

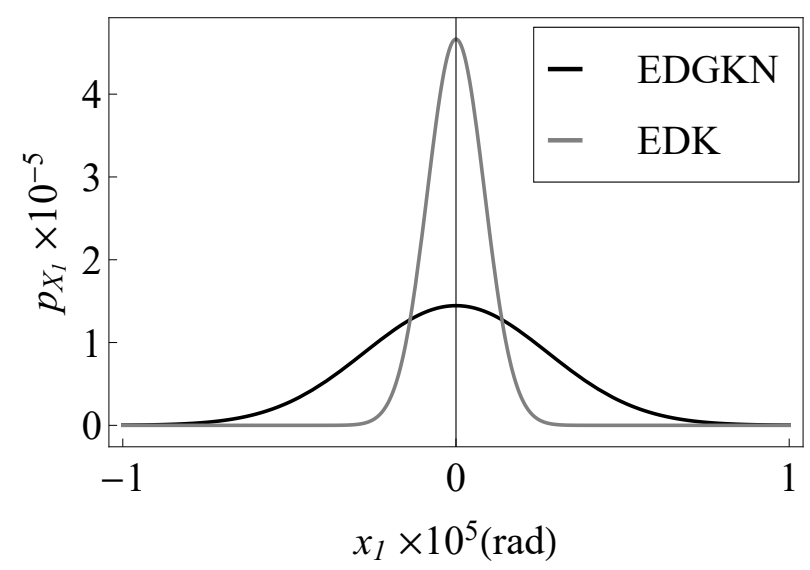

a)

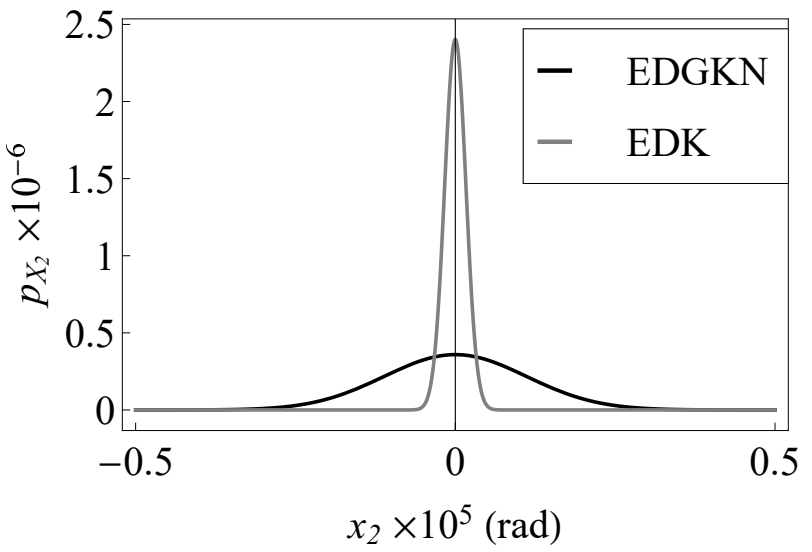

b)

FIGURE 3 Marginal PDFs $p_{X_{1}}$ and $p_{X_{2}}$ of the SEMRECs.

Performing the deviation comparison for the corresponding SEMRECs (12) and (14) with the intensity vector $\delta=$ $[0,0,0.1,0]^{\top} \mathrm{s}^{-2}$, one gets the stochastic-excitation ratios

$$
\rho_{1}=3.2271>1 \quad, \quad \rho_{2}=6.6971>1 .
$$

Therefore, the stochastically reinforced self-excitation is effective for both state variables. The marginal PDF plots also confirm that in the EDGKN case the state variables distribute in much wider ranges (Fig. 3a, 3b). To further illustrate the mechanism of brake squeal, the time responses of the two SEMRECs to a specific excitation are calculated using the Euler-Maruyama method. It can be seen clearly in Fig. 4 that the vibration in the EDGKN case are much larger so it is more likely to produce brake squeal than in the EDK case. The frequency characteristic of the responses $X_{1 t}$ and $X_{2 t}$ for the EDGKN case is visualized by the plot of the absolute values of corresponding transfer functions $H_{1}(f)$ and $H_{2}(f)$ in Fig. 5, the peaks of the plots lie in the kHz-range, fitting the characteristic of brake squeal. The contour plot in Fig. 6 shows that the dominating frequencies are nearly independent on the disk speed, which is a typical property of disk brake squeal ${ }^{[24]}$ as illustrated by measurement results in Fig. 7 (taken from $\stackrel{[18]}{ }$, Fig. 42).

This case study shows that, though still having a negative maximum real part of the eigenvalues, an EDGKN system with stochastic excitation may exhibit a vibration that has some similar properties to a measured vibration during brake squeal. It means stochastically reinforced self-excitation should be considered additionally to the well-known maximum real part of the eigenvalues when analyzing brake squeal through mathematically models. 


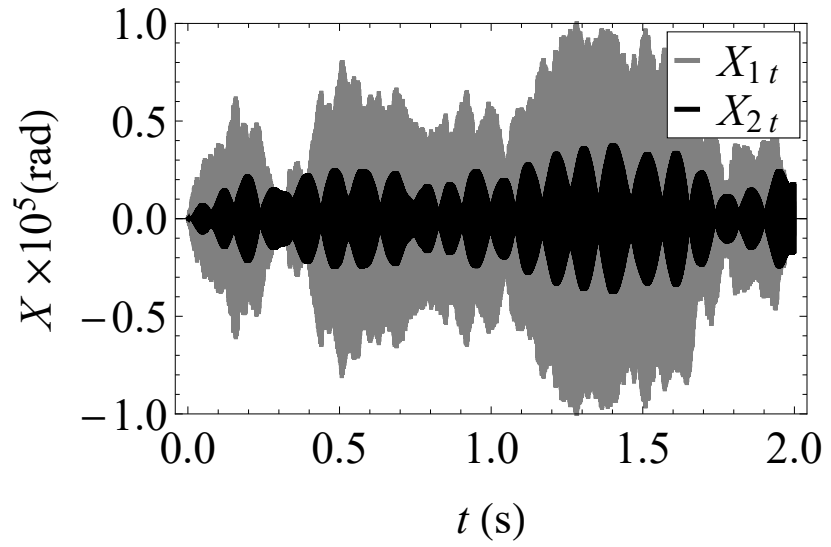

a) EDGKN system

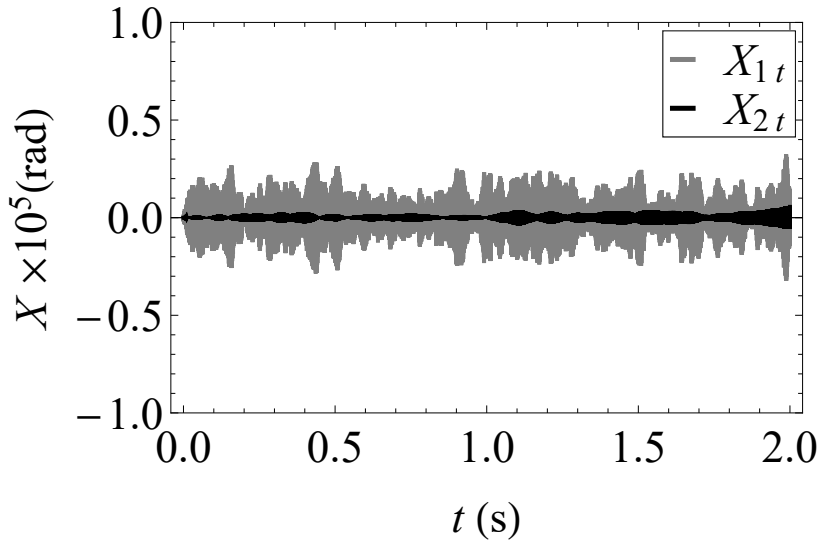

b) EDK system

FIGURE 4 Realizations of the processes $X_{1 t}$ and $X_{2 t}$ for the SEMRECs with the same excitation intensity

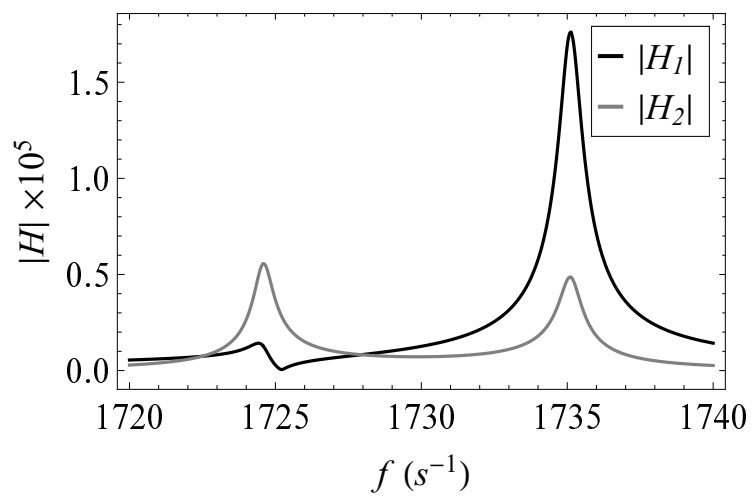

FIGURE 5 Transfer functions $\left|H_{1}(f)\right|$ and $\left|H_{2}(f)\right|$ of the EDGKN system

\section{2 | Parameter study}

It is complicated to do a full parameter study for the EDGKN system, since the number of parameters is as high as 8 and the number of physical parameters is even higher. To simplify the problem, the number of parameters should be reduced.

Starting from the matrices (27)-(30), doing a scale in time and simplifying the numeric values, one obtains the following dimensionless matrices

$$
\begin{gathered}
\mathbf{D}=\left[\begin{array}{cc}
0.0008 & 0 \\
0 & 0.00006
\end{array}\right], \\
\mathbf{G}=\left[\begin{array}{cc}
0 & 0.003 \\
-0.003 & 0
\end{array}\right], \\
\mathbf{K}=\left[\begin{array}{cc}
1 & -0.0006 \\
-0.0006 & 0.99
\end{array}\right], \\
\mathbf{N}=\left[\begin{array}{cc}
0 & 0.0006 \\
-0.0006 & 0
\end{array}\right],
\end{gathered}
$$

The considered intensity vector is $\delta=[0,0,1,0]^{\top}$.

Since the gyroscopic terms are of interest, matrix G should be parameterized 


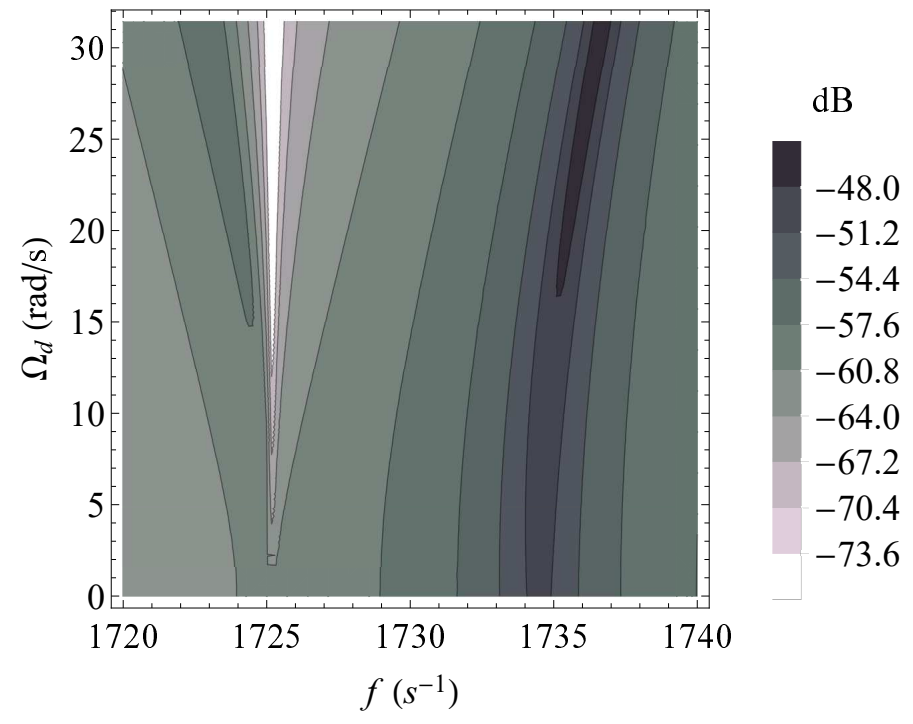

FIGURE 6 Transfer function $\left|H_{1}(f)\right|$ of the EDGKN system at different disk speeds.

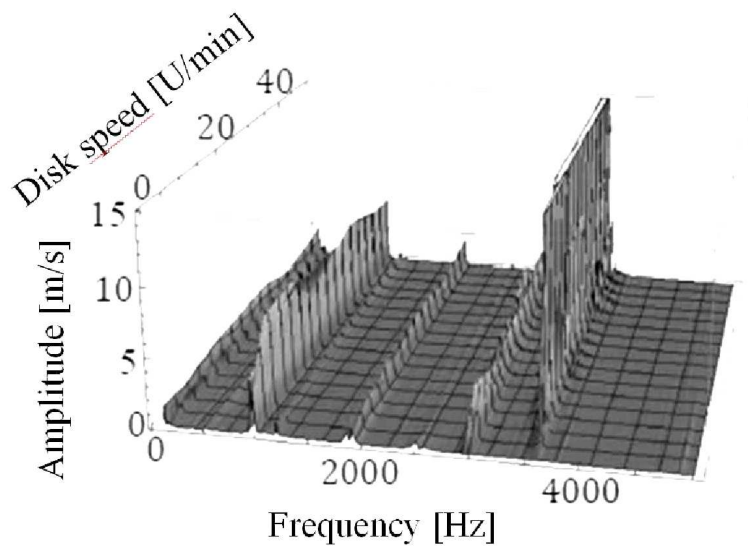

FIGURE 7 Measured results from a brake test rig. Vibration amplitude of the disk (squeal intensity) over the frequency and the rotational speed of the disk (taken from $\stackrel{[18]}{ }$, Fig. 42)

$$
\mathbf{N}=\left[\begin{array}{cc}
0 & 0.0008 \gamma \\
-0.0008 \gamma & 0
\end{array}\right],
$$

where $\gamma$ is defined as the ratio between the second element of the first row of $\mathbf{G}$ and the first element of $\mathbf{D}$

$$
\gamma=\frac{g_{12}}{d_{11}}
$$

The matrix $\mathbf{D}$ is also parameterized

$$
\mathbf{N}=\left[\begin{array}{cc}
0.0008 & 0 \\
0 & 0.0008 \beta
\end{array}\right],
$$

where $\beta$ is defined as the ratio between the damping coefficient in the second equation and that in the first one 


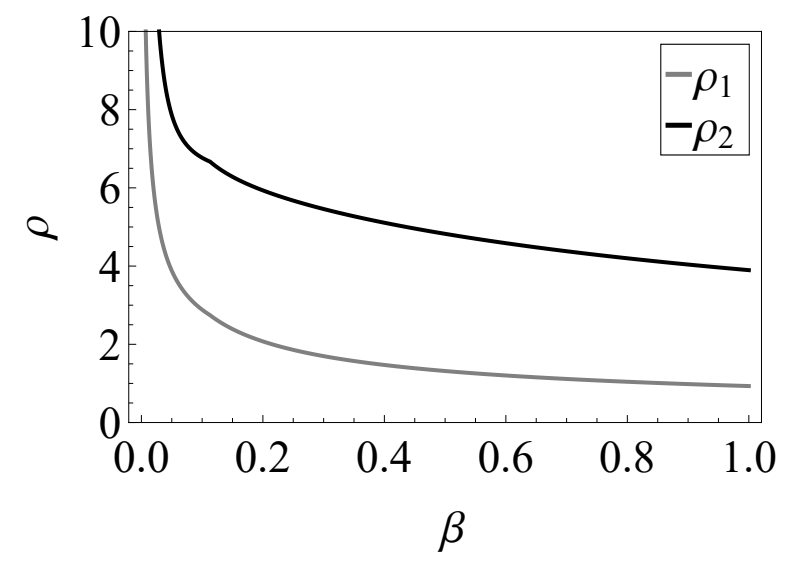

FIGURE 8 Stochastic-excitation ratios dependence on $\beta$.

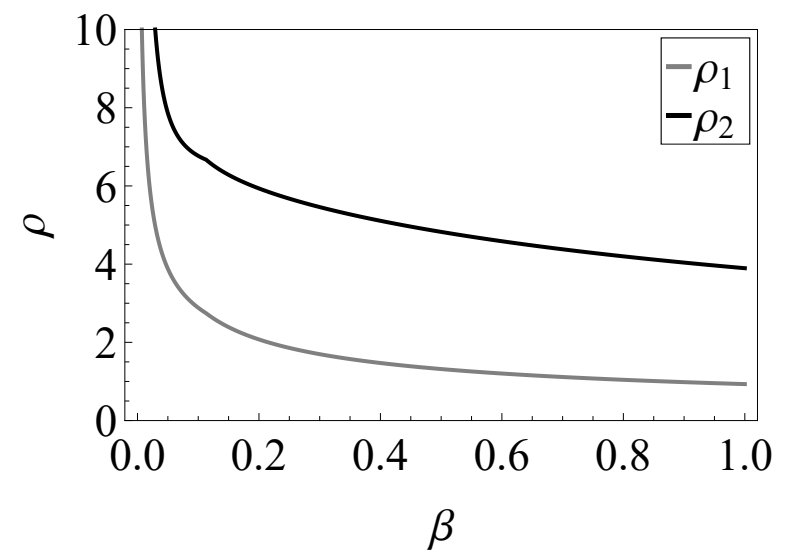

FIGURE 9 Stochastic-excitation ratios dependence on $\gamma$.

$$
\beta=\frac{d_{22}}{d_{11}} .
$$

When not otherwise indicated, the parameters are set as $\beta=3.7$ and $\gamma=0.075$.

It can be seen in Fig. 8 that as $\beta$ becomes lower, both the stochastic-excitation ratios increase significantly. Taking a look back at (5), this means lowering $d_{t}$ - the rotational damping of the wobbling disk - may increase the intensity of the resulting vibrations.

The dependence of the stochastic-excitation ratios on $\gamma$ is shown in Fig. 9. When $\gamma$ reaches 4.2, $\rho_{1}$ peaks at 37 and $\rho_{2}$ at 11 . Considering (5), (8), (10) and (37), $\gamma$ and the disk speed $\Omega_{d}$ have a direct monotonic relationship so there is no upper bound for $\gamma$; however, if $\gamma$ is too high, the original EDGKN system is unstable so the deviation comparison is no longer valid. The stochastic-excitation ratios decrease when $\gamma$ increases beyond 4.2 but do not drop below 6 .

Fig. 10 shows the dependence of the stochastic-excitation ratios on both $\gamma$ and $\beta$, where $\beta$ takes all positive values below 1 and $\gamma$ varies from 0 to 15 . For all values of $\gamma$, the stochastic-excitation ratios increase rapidly when $\beta$ approaches 0 , just similar to the case shown in Fig. 8.

\section{5 | CONCLUSIONS AND OUTLOOK}

In this paper a stochastically excited EDGKN system derived from a 2-DOF model for brake squeal has been proved to possibly have much larger vibration than an EDK system with the same negative maximum real part of the eigenvalues and under the 


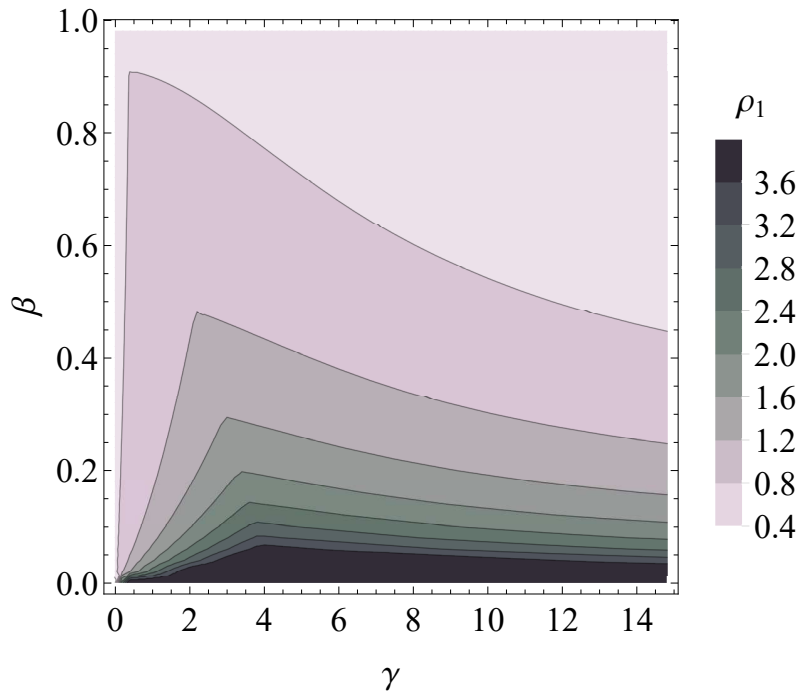

a)

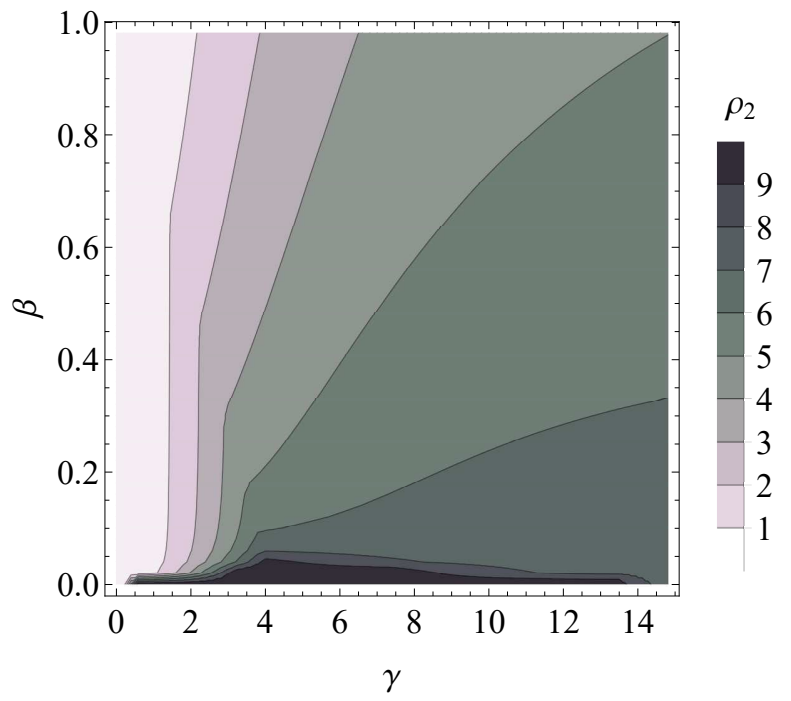

b)

FIGURE 10 Stochastic-excitation ratios with varied $\gamma$ and $\beta$ for a) the first state variable and $b$ ) the second state variable .

same stochastic excitation. It means that when considering brake vibrations based on linear analysis, one should consider not only the stability of the system but also hypothesized stochastically reinforced self-excitation. A partial parameter study has shown that the ratio between the main damping coefficients and the gyroscopic terms do affect the stochastic-excitation ratios. Thus, future work might focus on the optimization to avoid brake squeal. Further parameter study might also be performed to contribute more understanding to the stochastically excited EDGKN system.

Beyond stable linear systems, the combination and interaction of stochastically reinforced self-excitation, instability, and nonlinearities might be examined in future work.

\section{ACKNOWLEDGMENTS}

At the early stage of this study, the first author acknowledged the financial support by Project 911 (from Vietnamese Government) and a supplemental scholarship from DAAD.

\section{CONFLICT OF INTEREST}

The authors declare no potential conflict of interests.

\section{References}

[1] N.-T. Minh-Tuan, P. Wulff, N. Gräbner, U. von Wagner, On the influence of external stochastic excitation on linear oscillators with subcritical selfexcitation applied to brake squeal, in Theoretical Approaches in Non-Linear Dynamical Systems (DSTA 2019), (Eds.: J. Awrejcewicz, M. Kaźmierczak, J. Mrozowski ), Lodz University of Technology, 2019, pp. 361-370.

[2] N. M. Kinkaid, Oliver O'Reilly, P. Papadopoulos, Automotive disc brake squeal, Journal of Sound and Vibration 2003, 267, 105, doi:10.1016/S0022460X(02)01573-0.

[3] C. Cantoni, R. Cesarini, G. Mastinu, G. Rocca, R. Sicigliano, Brake comfort - A review 2009 47(8), 901, doi:10.1080/00423110903100432.

[4] K. Popp, M. Rudolph, Matthias Kröger, M. Lindner, Mechanisms to generate and to avoid friction induced vibrations, VDI Berichte 2002 , 1.

[5] T. Hamabe, I. Yamazaki, K. Yamada, H. Matsui, S. Nakagawa, M. Kawamura, Study of a Method for Reducing Drum Brake Squeal 1999, 108, 523, doi:10.4271/1999-01-0144. 
[6] U. von Wagner, D. Hochlenert, P. Hagedorn, Minimal models for disk brake squeal, Journal of Sound and Vibration 2007, 302, 527-539, doi:10.1016/j.jsv.2006.11.023.

[7] D. Hochlenert, U. von Wagner, How Do Nonlinearities Influence Brake Squeal?, SAE Technical Papers 2011, doi:10.4271/2011-01-2365.

[8] S. Koch, N. Graebner, H. Gödecker, U. Wagner, Nonlinear Multiple Body Models for Brake Squeal, PAMM 2017, 17, 33, doi:10.1002/pamm.201710010.

[9] Sebastian Kruse, Merten Tiedemann, Bernhard Zeumer, Pascal Reuss, Hartmut Hetzler, Norbert Hoffmann, The influence of joints on friction induced vibration in brake squeal, Journal of Sound and Vibration 2015, 340, doi:10.1016/j.jsv.2014.11.016.

[10] Yusuke Aoki, Yukio Nishizawa, Yutaka Kurita, Yasunori Oura, Friction Coefficient Variation at Minute Vibrating Velocity between Brake Pad and Disc Rotor, SAE Technical Papers 2013, 8, doi:10.4271/2013-01-2041.

[11] Yasuyuki K., Yusuke A., Yukio N., Evaluation of an Energy Dissipation Mechanism by Friction for Brake Shims, in SAE Technical Paper, SAE International, 2017. https://doi.org/10.4271/2017-01-2487

[12] N.-T. Minh-Tuan, Effect of vibrations on friction in the context of brake squeal, Doctoral thesis, Technische Universität Berlin, Berlin, 2019. http://dx.doi.org/10.14279/depositonce-8186

[13] Fabian Waleffe, Transition in shear flows. Nonlinear normality versus non-normal linearity, Physics of Fluids - PHYS FLUIDS 1995, 7, 3060, doi: $10.1063 / 1.868682$

[14] C. D. Cantwell, D. Barkley, H. M. Blackburn, Transient growth analysis of flow through a sudden expansion in a circular pipe, Physics of Fluids 2010 22(3), 034101, doi:10.1063/1.3313931.

[15] N. Hoffmann, L Gaul, Non-conservative beating in sliding friction affected systems: Transient amplification of vibrational energy and a technique to determine optimal initial conditions, Mechanical Systems and Signal Processing - MECH SYST SIGNAL PROCESS 2004, 18, 611, doi:10.1016/S08883270(03)00072-4.

[16] N. Hoffmann, Transient Growth and Stick-Slip in Sliding Friction, Journal of Applied Mechanics-transactions of The Asme - J APPL MECH 2006, 73, doi:10.1115/1.2165233

[17] N. Hoffmann, U. Stolz, On transient growth of wear pattern properties, Wear 2010, 268, 886, doi:10.1016/j.wear.2009.12.030.

[18] N. Gräbner, Analyse und Verbesserung der Simulationsmethode des Bremsenquietschens, Doctoral thesis, Technische Universität Berlin, Berlin, 2016. http://dx.doi.org/10.14279/depositonce-5577

[19] S. Schlagner, U. von Wagner, Quietschen von KFZ-Scheibenbremsen, PAMM 2006, doi:10.1002/pamm.200610146.

[20] D. Hochlenert, P. Hagedorn, Control of disc brake squeal - Modelling and experiments, Structural Control and Health Monitoring 2006, doi:10.1002/stc. 138

[21] P. Hagedorn, E. Heffel, P. Lancaster, P.C. Müller, S. Kapuria, Some recent results on MDGKN-systems, ZAMM - Journal of Applied Mathematics and Mechanics / Zeitschrift für Angewandte Mathematik und Mechanik 2015 95(7), 695, doi:10.1002/zamm.201300270.

[22] T. T. Soong, Random differential equations in science and engineering, Academic Press New York, New York, 1973.

[23] W. Martens, On the solution of the Fokker-Planck-Equation for multi-dimensional nonlinear mechanical systems, Doctoral thesis, Technische Universität Berlin, Berlin, 2014.

[24] Andrew Day in Braking of Road Vehicles, Andrew Day (Ed.), Butterworth-Heinemann, Oxford, 2014, pp. 343 - 384. 